Pacific Journal of Mathematics

ERGODIC AUTOMORPHISMS AND AFFINE
TRANSFORMATIONS OF LOCALLY COMPACT GROUPS m. 


\title{
ERGODIC AUTOMORPHISMS AND AFFINE TRANSFORMATIONS OF LOCALLY COMPACT GROUPS
}

\author{
M. RAJAgOpalan AND B. Schreiber
}

It has been conjectured that if a locally compact group $G$ has a continuous automorphism which is ergodic with respect to Haar measure then $G$ must be compact. This is true when $G$ is commutative or connected. In this paper further results in support of this conjecture are presented. In particular, it is shown that the problem can be reduced to the consideration of compactly generated, totally disconnected, locally compact groups without compact, open, normal subgroups and that the conjecture holds for many automorphisms of a certain class of such groups. Finally, the structure of locally compact groups which admit ergodic affine transformations is investigated.

The question of the existence of ergodic automorphisms on noncompact groups was first raised by P. Halmos [5, p. 29]. The commutative case was studied in [10] and [15] and the connected case in [8] and [14]. Theorem 1.1 below has been announced without proof in [16], and some of the results in $\S 2$ have been obtained independently and by somewhat different methods by R. Sato [11], [12] and by N. Aoki and Y. Ito [1].

1. Automorphisms. Let $G$ be a locally compact group and $T$ an ergodic (whence bi-continuous and measure preserving, as shown in [10]) automorphism of $G$. Thus, if $\lambda$ denotes a left Haar measure on $G, \lambda(A)=0$ or $\lambda\left(A^{c}\right)=0$ for any measurable subset $A$ of $G$ such that $T(A)=A$. Let $G_{0}$ denote the identity component of $G$.

THEOREM 1.1. If $G / G_{0}$ is compact then $G$ must be compact. Thus if there exists a noncompact group with an ergodic automorphism then there exists a noncompact totally disconnected one.

Proof. If $G / G_{0}$ is compact there is a unique maximal normal compact subgroup $N$ of $G$ such that $G / N$ is a Lie group [9, p. 175], [6, XV.3]. Since $N$ must be invariant under $T$, there is induced an ergodic automorphism $\widetilde{T}$ of $G / N$ (cf. [8]). Since $(G / N)_{0}$ is open and invariant under $\widetilde{T}, G / N$ is in fact connected, whence compact. Thus $G$ is compact.

If $G$ is a noncompact group with ergodic automorphism $T$, then by the above the totally disconnected group $G / G_{0}$ must be noncompact, 
and it possesses the ergodic automorphism $\widetilde{T}$ induced by $T$ as above.

For the remainder of this section $G$ will be assumed to be nondiscrete and totally disconnected with ergodic automorphism $T$.

THeorem 1.2. Suppose that $G$ satisfies one of the following conditions:

(i) Every compact subset of $G$ is contained in a compact subgroup of $G$.

(ii) $G$ has a compact, open, normal subgroup. Then $G$ must be compact.

Proof. To prove that (i) implies compactness of $G$ it clearly suffices to prove that $G$ must be compactly generated. Let $H$ be a compact, open subgroup of $G$, and for each $n$ let $K_{n}$ be the group generated by $H \cup T(H) \cup \cdots \cup T^{n}(H)$. Then $K_{1} \subset K_{2} \subset \cdots$ and $T\left(K_{n}\right) \subset K_{n+1}, n=1,2, \cdots$. Set $K=\bigcup_{n=1}^{\infty} K_{n}$, so that $K$ and $T(K)$ are open subgroups of $G$ with $T(K) \subset K$.

Suppose $T(K) \neq K$, and let $S=K \backslash T(K)$. Note that

$$
T^{m}(S) \cap T^{n}(S)=\varnothing
$$

if $m \neq n$, and $\lambda(S)>0$. Choose $P \subset S$ with $\lambda(P)>0$ and $\lambda(S \backslash P)>0$ and set $Q=\bigcup_{n=-\infty}^{\infty} T^{n}(P)$. Then $T(Q)=Q$ and $Q \cap(S \backslash P)=\varnothing$, contradicting the ergodicity of $T$. Thus $T(K)=K$, so $K=G$ since $K$ is closed. Hence $T^{-1}(H) \subset K_{n}$ for some $n$, whence $T^{-1}\left(K_{n}\right) \subset K_{n}$. Repeating the argument above we obtain $T^{-1}\left(K_{n}\right)=K_{n}$, so $G=K_{n}$ is compactly generated.

If (ii) holds, let $H$ be a compact, open, normal subgroup of $G$ and let the $K_{n}$ be as defined above. Then each $K_{n}=H \cdot T(H) \cdots T^{n}(H)$ is compact, so the argument above shows $G$ is compact.

COROLlaRY 1.3. If $G$ is the union of an increasing sequence of compact open subgroups, then $G$ is compact.

COROLLARY 1.4. If $G$ is nilpotent, then it must be compact.

Proof. The proof is by induction on the length of the ascending central series of $G,\{e\} \subset Z(G) \subset Z^{2}(G) \subset \cdots \subset Z^{n}(G)=G$. If $n=1$, then $G$ is abelian, whence compact. In general, since $Z(G)$ is invariant under $T, G / Z(G)$ has an ergodic automorphism (cf. Theorem 1.1) and $Z^{n-1}(G / Z(G))=G / Z(G)$. If we assume that this implies $G / Z(G)$ is compact, we may invoke the main theorem of [4] to conclude that (ii) of Theorem 1.2 holds, whence $G$ is compact, and the induction is complete.

CoRollary 1.5. If $G$ is maximally almost periodic (i.e., has 
sufficiently many finite-dimensional continuous unitary representations to separate points) then it must be compact.

Proof. Since we have shown $G$ is compactly generated, it is known that $G$, being totally disconnected, must satisfy (ii) of Theorem 1.2 [7, Corollary XII. 3, pp. 58-59].

Miscellaneous Remarks.

(i) $G$ must be unimodular. This follows either from a calculation which shows that the modular function, which is continuous, is invariant under $T$ or from the fact that the modular function is a homomorphism which must equal one on the finite collection of compact subgroups which generate $G$ as in the proof of Theorem 1.2.

(ii) In testing the validity of our conjecture it suffices to consider only metrizable groups. Indeed, if $G$ and $T$ are as above let $H$ be a compact normal subgroup of $G$ such that $G / H$ is metrizable. Let $K=\bigcap_{n=-\infty}^{\infty} T^{n}(H)$. Then $T(K)=K$, so $G / K$ has an ergodic automorphism and is metrizable.

(iii) If there exists an ergodic inner automorphism, $T(x)=a^{-1} x a$ for some $a \in G$, then $G$ must be noncompact and the subgroup of $G$ generated by a must be discrete (and infinite). For otherwise there is a compact subgroup $K$ of $G$ containing $a$. If $H$ is a compact open subgroup of $G$, then clearly $G=K H K$ and is thus compact. But then every neighborhood of the identity in $G$ contains a normal open subgroup, which is impossible.

We conclude this section by showing, in Theorem 1.8, that the conjecture in question holds for many automorphisms of a class of totally disconnected groups which do not, in general, satisfy (i) or (ii) of Theorem 1.2. We are grateful to A. Borel for his suggestions regarding the proof of Theorem 1.8 .

LEMMA 1.6. Let $H$ be a closed normal subgroup of $G$ such that $G / H$ is compact and $H$ is the union of an increasing sequence of compact open subgroups. Then $G$ is also the union of an increasing sequence of compact open subgroups.

Proof. Let $H_{1} \subset H_{2} \subset \cdots \subset H$ as in our hypothesis, and choose a symmetric compact subset $K$ of $G$ such that $G=H K$. Since $K^{2}=K \cdot K$ is compact, it is contained in $H_{n} K$ for some $n$. Then

$$
K^{3} \subset H_{n} K^{2} \subset H_{n}^{2} K=H_{n} K,
$$

and so on. Thus, denoting by $[K]$ the subgroup generated by $K$,

$$
[K]=\bigcup_{m=1}^{\infty} K^{m} \subset H_{n} K,
$$


so $[K]^{-}$is compact. Hence we may assume $K$ is a group.

Let $G_{n}$ be the closed subgroup generated by $H_{n}$ and $K$, $n=1,2, \cdots$. Then $G=\bigcup_{n=1}^{\infty} G_{n}$, so by the Baire category theorem $G_{n}$ is open for all sufficiently large $n$. To show each $G_{n}$ is compact, notice that, given $n$, we can find a positive integer $m$ such that $h^{x}=x h x^{-1} \in H_{m}$ for all $h \in H_{n}$ and $x \in K$. It follows that $\left[H_{n}, K\right] \subset H_{m} K$, for every element of $\left[H_{n}, K\right]$ is of the form

$$
h_{1} x_{1} \cdots h_{r} x_{r}=h_{1} h_{2}^{x_{1}} h_{3}^{x_{1} x_{2}} \cdots h_{r}^{x_{1}} \cdots x_{r} x_{1} \cdots x_{r}\left(h_{i} \in H_{n}, x_{i} \in K\right)
$$

and hence lies in $H_{m} K$.

COROLlary 1.7. If $G$ has a normal series consisting of closed subgroups whose successive quotients are all unions of increasing sequences of compact open subgroups, then $G$ is also the union of such an increasing sequence.

Proof. We have $\{e\}=G_{0} \subset G_{1} \subset \cdots \subset G_{n}=G$, and we apply induction on $n$. The case $n=1$ leaves nothing to prove; in general $G_{n-1}$ is normal in $G$. Let $\pi: G \rightarrow G / G_{n-1}$ be the canonical map, and write $G / G_{n-1}=H_{1} \cup H_{2} \cup \cdots$, the union of an increasing sequence of compact open subgroups. By the induction hypothesis and Lemma 1.6 we may write $\pi^{-1}\left(H_{m}\right)$ as the union of such an increasing sequence:

$$
\pi^{-1}\left(H_{m}\right)=\bigcup_{k=1}^{\infty} H_{m k}, \quad m=1,2, \cdots,
$$

and by choosing subsequences we may assume $H_{m+1, k} \supset H_{m k}$ for all $k$ and $m$. Let us now choose a sequence $\left\{G_{m}\right\}$ of compact open subgroups of $G$ as follows. Let $G_{1}^{\prime}=H_{11}$, and suppose $G_{1}, \cdots, G_{m}^{\prime}$ have been chosen. Let $G_{m+1}^{0}=H_{m+1 k}$, $k$ being so large that $G_{m+1}^{3} \supset G_{m}^{3} \cup H_{m m}$. Then the $G_{m}^{0}$ are increasing, and it is clear that

$$
\bigcup_{m=1}^{\infty} G_{m} \supset \bigcup_{m=1}^{\infty} \bigcup_{k=1}^{\infty} H_{m k}=G \text {. }
$$

Let $k$ be a nondiscrete, locally compact, totally disconnected field. If $G$ is a connected (in the Zariski topology) linear algebraic group defined over $k$ ( $k$-group) [2], [3], $G(k)$ will denote the rational elements of $G$ over $k$ and $\mathrm{Aut}_{k} G$ the group of automorphisms of $G$ defined over $k$. The restriction to $G(k)$ of any $T \in A u_{k} G$, denoted also by $T$, is a topological automorphism of $G(k)$ when $G(k)$ is given its locally compact topology obtained by realizing it as a subgroup of $G L(n, k)$ for some $n$. Let $R$ be the radical of $G$ and $U$ the unipotent radical. Recall that $R[U]$ is the unique maximal connected, solvable [unipotent], Zariski-closed, normal subgroup of $G$. If $k$ is of characteristic zero, 
then $U$ (whence also $R$ ) is always defined over $k$. However if $k$ has positive characteristic this need not be the case.

THEOREM 1.8. Let $G$ be a connected linear algebraic group defined over the nondiscrete, locally compact, totally disconnected field $k$ such that $U$ is defined over $k$, and let $T \in$ Aut $_{k} G$. If $T$ is ergodic on $G(k)$, then $G(k)$ is compact and solvable. In fact, if char $k=0$ then $G$ is an anisotropic torus.

Proof. Since $R / U$ is the (Zariski-) connected component of the identity of the center of $G / U$ [3, Proposition 11.21], $R$ is defined over $k$. Let $G^{\prime}=G / R$ and $\pi: G \rightarrow G / R$ the canonical map. $G^{\prime}$ is connected, defined over $k$, and semi-simple. And $T(R)=R$, so $T$ induces an automorphism $T^{\prime}$ of $G^{\prime}$, defined over $k$, whose restriction to $\pi(G(k))=G(k) / R(k)$ is an ergodic automorphism. Since $G^{\prime}$ is semisimple, Aut $G^{\prime}$ is a k-group, and we have (Aut $\left.G^{\prime}\right)(k)=\operatorname{Aut}_{k} G^{\prime}$. Furthermore the semi-direct product $H=G^{\prime} \cdot\left(\right.$ Aut $G^{\prime}$ ) is also a $k$-group, so $H(k)=G^{\prime}(k) \cdot\left(\operatorname{Aut}_{k} G^{\prime}\right)$ may be realized as a subgroup of $G L(n, k)$ for some $n$. Let $H(k)$ be so realized. Then $T^{\prime}$ is given on $G(k)$ by conjugation by some element of $G L(n, k)$. Given $\lambda \in k$, consider the continuous mapping $\varphi_{2}: \pi(G(k)) \rightarrow k$ given by

$$
\varphi_{\lambda}(x)=\operatorname{det}(x-\lambda e) .
$$

$\varphi_{2}$ is invariant under $T^{\prime}$, whence constant on $\pi(G(k))$. Thus

$$
\varphi_{\lambda}(x)=\varphi_{\lambda}(e)=(1-\lambda)^{n}, \quad \lambda \in k, x \in \pi(G(k)) .
$$

Hence every element of $\pi(G k))$ has as characteristic polynomial $(1-\lambda)^{n}$, i.e., $\pi(G(k))$ consists of unipotent matrices.

Recall that $G(k)$ has a natural structure as a $k$-analytic variety of analytic dimension equal to the dimension of $G$ [13, Appendix III]. Since $G$ is connected $G(k)$ cannot be contained in any proper analytic subset of $G$. Thus $G(k)$ is Zariski-dense in $G$, so $\pi(G(k))$ is dense in $G^{\prime}$. Thus $G^{\prime}$ is both unipotent and semi-simple, whence trivial.

Thus $G=R$ is solvable, so $G / U$ is a torus [3, Theorem 10.6], whence abelian. As above $T$ induces an ergodic automorphism of $G(k) / U(k)$, so $G(k) / U(k)$ is compact. Moreover, $U$ is trigonalizable over $k$ [3, Corollary 15.5], and $k$ is the union of an increasing sequence of compact open subgroups. By Lemma 1.6 and Corollary 1.7 we conclude that $U(k)$ is the union of such a sequence, and hence so is $G(k)$. Corollary 1.3 then implies $G(k)$ is compact.

If $k$ is of characteristic zero, whence perfect, and if $U$ were nontrivial, then $U(k)$ would have a normal series of closed subgroups whose successive quotients are isomorphic to the additive group of $k$. But $G(k)$ being compact, this is impossible, and it follows that $G$ is 
an anisotropic torus.

REMARK. Using some deeper results from the theory of algebraic groups, Theorem 1.8 can be generalized in several directions. These generalizations will be presented elsewhere.

2. Affine transformations. Let $G$ be a locally compact group and $T$ an affine transformation of $G$. That is, $T(x)=a \tau(x), x \in G$, for some continuous automorphism $\tau$ of $G$ and $a \in G$. As in $\S 1$, we are interested in structural results about $G$ implied by the assumption that $T$ is ergodic. We no longer expect that $G$ must be compact; for example, $T(x)=x+1$ is ergodic on $Z$.

The proofs of our first two lemmas are analogous to those of the corresponding facts about automorphisms found in [8] and [10].

LEMma 2.1. If $T$ is ergodic then it must be bicontinuous and measure preserving.

Lemma 2.2. Let $H$ be a closed normal subgroup of $G$ such that $\tau(H)=H$, and let $\widetilde{\tau}$ denote the induced automorphism of $G / H$. If $T$ is ergodic on $G$ then the affine transformation $\widetilde{T}(\bar{x})=\bar{a} \widetilde{\tau}(\bar{x})$ is ergodic on $G / H$.

Lemma 2.3. Let $G$ be discrete and $T$ ergodic on $G$. Then $G$ is finitely generated.

Proof. A computation shows that for $n>0$,

(1) $T^{n}(e)=a \tau(a) \cdots \cdot \tau^{n-1}(a)$,

(2) $T^{-n}(e)=\left(\tau^{-n}(a) \tau^{-(n-1)}(a) \cdots \tau^{-1}(a)\right)^{-1}=\left(\tau^{-n}\left(T^{n}(e)\right)\right)^{-1}$.

There exists $p \in Z$ such that $T^{p}(e)=\tau(a)$. Clearly $p=0$ (i.e., $T$ an automorphism) is impossible, while $p=1$ gives $T\left(a^{n}\right)=a^{n+1}$ and this is the example cited above.

Suppose $p>1$. Then from (1) we have

$$
\tau^{p-1}(a)=\left(a \tau(a) \cdots \tau^{p-2}(a)\right)^{-1} \tau(a),
$$

and it follows that

$$
T^{n}(e) \in\left[a, \tau(a), \cdots, \tau^{p-2}(a)\right]=H
$$

for all $n>0$. And applying $\tau^{-1}$ to (1) gives $\tau^{-1}(a) \in H$ and hence $T^{-n}(e) \in H, n>0$. Thus $G=$ orbit of $e=H$ is finitely generated.

Now assume $p<0$. Then by (2) we have

$$
T^{n}(e) \in\left[a, \tau^{-1}(a), \cdots, \tau^{p}(a)\right]=K, \quad n>0,
$$

while applying $\tau^{-1}$ to (2) shows $\tau^{p-1}(a) \in K$ and hence $T^{-n}(e) \in K, n>0$. 
Again $G$ is finitely generated.

THEOREM 2.4. If $G$ has an ergodic affine transformation, then $G$ is compactly generated. If $G$ has a nontrivial, compact, open, normal subgroup, then $G$ is compact.

Proof. If $G$ is discrete, then it is finitely generated by Lemma 2.3, so we assume $G$ is nondiscrete. Assume first that $G$ has a compact open subgroup $H$. Let $K_{n}$ be the group generated by

$$
\left\{a, \tau(a), \cdots, \tau^{n-1}(a)\right\} \cup H \cup \cdots \cup \tau^{n}(H), \quad n=1,2, \cdots .
$$

The proof that $G$ is compactly generated now proceeds like that of Theorem 1.2 (here $T(K)$ is an open left coset), except that we now choose $n$ so large that $\left\{\tau^{-1}(a)\right\} \cup \tau^{-1}(H) \subset K_{n}$.

Now let $G$ be any locally compact group with identity component $G_{0}$, and let $\pi: G \rightarrow G / G_{0}$ be the natural map. By Lemma 2.2 and the argument above there is a compact generating set $C$ for $G / G_{0}$. If $U$ is a compact neighborhood of the identity in $G$ such that $\pi(U) \supset C$, then $U \cap G_{0}$ generates $G_{0}$, and it is easy to see that $U$ generates $G$.

Suppose the $H$ chosen above is normal and nontrivial. Let

$$
H_{n}=\tau^{-n}(H) \cdots H \cdots \tau^{n}(H), \quad n=1,2, \cdots,
$$

and set $H^{*}=\bigcup_{n=1}^{\infty} H_{n}$, so that $\tau\left(H^{*}\right)=H^{*}$. Then by Lemma 2.2 $G / H^{*}$ is discrete and has an ergodic affine transformation. If $G / H^{*}$ is infinite then $T^{m}\left(H^{*}\right) \cap T^{n}\left(H^{*}\right)=\varnothing$ if $m \neq n$. But this clearly implies $H=H^{*}=\{0\}$, as in the proof of Theorem 1.2, a contradiction.

Thus $G / H^{*}$ is finite, and we have $T^{m}(a) \in H^{*}$ for some $m$. Let $A=\left\{e, a, \cdots, \tau^{m-1}(a)\right\}$. Replacing $H$ by $H_{n}$ for sufficiently large $n$, we may assume that $T^{m}(a) \in H$ and $A H$ is a subgroup of $G$ (see proof of Lemma 1.6). The argument above then shows

$$
G=K_{n}=A H \tau(H) \cdots \tau^{n}(H)
$$

for some $n$, so $G$ is compact.

COROLlaRY 2.5. If $G$ is maximally almost periodic and has an ergodic affine transformation, then $G$ is discrete or compact.

Proof. See Corollary 1.5.

Lemma 2.6. $R^{n}$ has no ergodic affine transformations.

Proof. (cf. [5, p. 28]) Induction on $n$. It is easy to see no affine transformation on $R$ is ergodic. Assume $R^{n-1}$ has no ergodic affine transformations, and let $T(x)=\tau(x)+a$ be affine on $R^{n}$. Let $\pi: R^{n} \rightarrow R^{n+1}$ be given by $\pi\left(x_{1}, \cdots, x_{n}\right)=\left(x_{1}, \cdots, x_{n}, 1\right)$, and let $S$ be 
the linear transformation of $R^{n+1}$ given by the matrix

$$
\left(\begin{array}{ll}
\tau & a \\
0 & 1
\end{array}\right),
$$

so that $S \circ \pi=\pi \circ T$. By Lemma 2.1 we have $\operatorname{det} S=\operatorname{det} \tau= \pm 1$. Suppose first that $\tau$ is unipotent. Then $\tau$ has an eigenvector in $R^{n}$, so we may apply Lemma 2.2 and the induction assumption to conclude that $T$ is not ergodic.

If $\tau$ is not unipotent we shall construct a nonconstant continuous function on the hyperplane $R^{n} \times\{1\}$ which is invariant under $S$, proving that $T$ is not ergodic. Consider $S^{*}$, which is given by the matrix

$$
\left(\begin{array}{ll}
\tau^{*} & 0 \\
a & 1
\end{array}\right)
$$

on the dual space $\left(R^{n+1}\right)^{*}$. Since $\tau$, whence $\tau^{*}$, is not unipotent, neither is $S^{*}$. Thus the complexification $S_{*}^{*}$ of $S^{*}$ has an eigenvalue $\lambda \neq 1$. The annihilator of $C^{n}$ in $\left(C^{n+1}\right)^{*}$ consists of eigenvectors of $S_{c}^{*}$ with eigenvalue 1 . It follows, then, that any eigenvector for $\lambda$ assumes arbitrarily large values on $R^{n} \times\{1\} \subset C^{n+1}$. Indeed, if $z(x)$ is such an eigenvector, then $z$ does not annihilate $C^{n}$, so being complex linear, it cannot annihilate $R^{n}$. Thus $z$ assumes arbitrarily large values on $R^{n}$, whence on $R^{n} \times\{1\}$. Let $z_{1}, \cdots, z_{k}$ denote eigenvectors corresponding to the distinct eigenvalues of $S_{c}^{*}$ whose respective multiplicities are $n_{1}, \cdots, n_{k}$, and set

$$
f(x)=\left|z_{1}(x)^{n_{1}} \cdots z_{k}(x)^{n_{k}}\right| \cdot
$$

Then $f \circ S=f$ and $f$ is not constant.

Lemma 2.7. Let $T$ be an ergodic affine transformation of $Z^{n}$. Then $n=1$ and $T(x)=x \pm 1$.

Proof. It is easy to see that if $n=1$ then $T(x)=x \pm 1$. The rest of the proof is by an induction similar to that of Lemma 2.6: $\tau$ is given by a matrix with integer coefficients and determinant \pm 1 . If $\tau$ is not unipotent the argument above shows that $T$ is not ergodic. (Note that $f$ assumes arbitrarily large values on $Z^{n} \times\{1\}$.) On the other hand, if $\tau$ is unipotent then it has an eigenvector over $Q$, whence also over $Z$. Let $x_{0} \in Z^{n}$ be an eigenvector and $H=Q x_{0} \cap Z^{n}$. By Lemma 2.2 the induced affine transformation of $Z^{n} / H \cong Z^{n-1}$ is ergodic. The lemma now follows by induction once we settle the case $n=2$. But in this case $Z^{2} / H \cong Z$ and the induced affine map is a translation. Whence the orbit of 0 under $T$ is a proper subset of $Z^{2}$, so $T$ is not ergodic. 
THEOREM 2.8. Let $G$ be abelian and have an ergodic affine transformation. Then $G=Z$ or $G$ is compact.

Proof. If $G$ is discrete and infinite, then by Lemma 2.3 and Theorem $2.4 G$ is finitely generated and torsion free, so Lemma 2.7 implies $G=Z$.

Suppose $G$ is nondiscrete, and let $G_{0}$ denote its identity component. Then $G / G_{0}$ must be finite or nondiscrete. For $G / G_{0}$ discrete and infinite implies $G / G_{0}=Z$, and this leads to a contradiction as in the proof of Theorem 2.4. If $G / G_{0}$ is nondiscrete, then by Theorem 2.4 $G / G_{0}$ is compact. Thus in general, $G=R^{n} \times H$ with $H$ compact. Since $H$ is invariant under any automorphism of $G$, it follows from Lemmas 2.2 and 2.6 that $n=0$ and $G=H$ is compact.

COROLLARY 2.9. If $G$ is nilpotent and has an ergodic affine transformation, then $G=Z$ or $G$ is compact.

Proof. The proof is analogous to that of Corollary 1.4 .

\section{REFERENCES}

1. N. Aoki and Y. Ito, Ergodic properties of affine transformations, J. Math. Anal., Appl., to appear.

2. A. Borel, Groupes linéaires algébriques, Ann. of Math. (2) 64 (1956), 20-80.

3. — Linear Algebraic Groups, W. A. Benjamin, New York, 1969.

4. S. Grosser and M. Moskowitz, On central topological groups, Trans. Amer. Math. Soc. 127 (1967), 317-340.

5. P. R. Halmos, Lectures on Ergodic Theory, Pub. Math. Soc. Japan, Kenkyusha, Tokyo, 1956.

6. G. Hochschild, The Structure of Lie Groups, Holden-Day, San Francisco, 1965.

7. K. H. Hoffman and P. S. Mostert, Splitting in Topological Groups, Mem. Amer. Math. Soc. No. 43, Providence, R.I., 1963.

8. R. Kaufman and M. Rajagopalan, On automorphisms of a locally compact group, Mich. Math. J., 13 (1966), 373-374.

9. D. Montgomery and L. Zippin, Topological Transformation Groups, Interscience, New York, 1955.

10. M. Rajagopalan, Ergodic properties of automorphisms of a locally compact group, Proc. Amer. Math. Soc., 17 (1966), 372-376.

11. R. Sato, On locally compact abelian groups with dense orbits under continuous affine transformations, Proc. Japan Acad., 46 (1970), 147-150.

12. - Properties of ergodic affine transformations of locally compact groups. II, Proc. Japan Acad., 46 (1970), 236-238.

13. A. Weil, Foundations of Algebraic Geometry, 2nd ed., Amer. Math. Soc. Colloquium Pub. No. 29, Providence, R. I., 1962.

14. T. S. Wu, Continuous automorphisms on locally compact groups, Math. Z., 96 (1967), 256-258.

15. S. A. Yuzvinskii, Metric properties of automorphisms of locally compact commutative groups, Sibirsk. Mat. Zh., 6 (1965), 244-247. 
16. Metric properties of automorphisms of a locally compact group, Russ. Math. Surveys, 22 (1967), 47-52.

Received July 6, 1970. The second author was partially supported by NSF Grant GP-13741.

UNIVERSITY OF ILLINOIS

AND

Madurai University, Madurai, India

WAYNe STATE University 


\section{PACIFIC JOURNAL OF MATHEMATICS}

\section{EDITORS}

H. SAMELSON

Stanford University

Stanford, California 94305

C. R. Hовву

University of Washington

Seattle, Washington 98105
J. DUGUNDJI

Department of Mathematics

University of Southern California

Los Angeles, California 90007

RICHARD ARENS

University of California

Los Angeles, California 90024

\section{ASSOCIATE EDITORS}
E. F. BECKENBACH
B. H. NeumanN
F. WOLF
K. YoSHIDA

\section{SUPPORTING INSTITUTIONS}

UNIVERSITY OF BRITISH COLUMBIA

CALIFORNIA INSTITUTE OF TECHNOLOGY

UNIVERSITY OF CALIFORNIA

MONTANA STATE UNIVERSITY

UNIVERSITY OF NEVADA

NEW MEXICO STATE UNIVERSITY

OREGON STATE UNIVERSITY

UNIVERSITY OF OREGON

OSAKA UNIVERSITY

UNIVERSITY OF SOUTHERN CALIFORNIA
STANFORD UNIVERSITY

UNIVERSITY OF TOKYO

UNIVERSITY OF UTAH

WASHINGTON STATE UNIVERSITY

UNIVERSITY OF WASHINGTON

AMERICAN MATHEMATICAL SOCIETY CHEVRON RESEARCH CORPORATION NAVAL WEAPONS CENTER 


\section{Pacific Journal of Mathematics}

\section{Vol. 38, No. $1 \quad$ March, 1971}

Bruce Alan Barnes, Banach algebras which are ideals in a Banach algebra ..... 1

David W. Boyd, Inequalities for positive integral operators............... 9

Lawrence Gerald Brown, Note on the open mapping theorem .............. 25

Stephen Daniel Comer, Representations by algebras of sections over Boolean

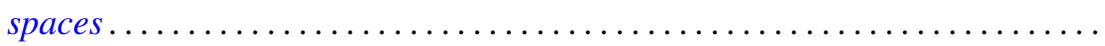

John R. Edwards and Stanley G. Wayment, On the nonequivalence of

conservative Hausdorff methods and Hausdorff moment sequences ........

P. D. T. A. Elliott, On the limiting distribution of additive functions $(\bmod 1) \ldots \ldots$

Mary Rodriguez Embry, Classifying special operators by means of subsets

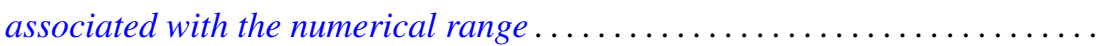

Darald Joe Hartfiel, Counterexamples to a conjecture of G. N. de Oliveira ......

C. Ward Henson, A family of countable homogeneous graphs...............

Satoru Igari and Shigehiko Kuratsubo, A sufficient condition for

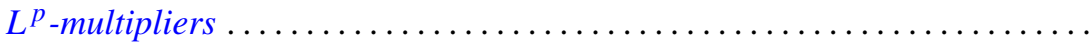

William A. Kirk, Fixed point theorems for nonlinear nonexpansive and

generalized contraction mappings............................

Erwin Kleinfeld, A generalization of commutative and associative rings ...... 95

D. B. Lahiri, Some restricted partition functions. Congruences modulo $11 \ldots \ldots 103$

T. Y. Lin, Homological algebra of stable homotopy ring $\pi *$ of spheres ....... 117

Morris Marden, A representation for the logarithmic derivative of a meromorphic function...........................

John Charles Nichols and James C. Smith, Examples concerning sum properties for metric-dependent dimension functions . .

Asit Baran Raha, On completely Hausdorff-completion of a completely

Hausdorff space.

M. Rajagopalan and Bertram Manuel Schreiber, Ergodic automorphisms and affine transformations of locally compact groups..........

N. V. Rao and Ashoke Kumar Roy, Linear isometries of some function

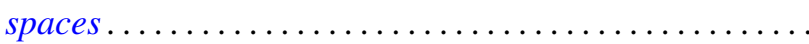

William Francis Reynolds, Blocks and F-class algebras of finite groups

Richard Rochberg, Which linear maps of the disk algebra are multiplicative ...

Gary Sampson, Sharp estimates of convolution transforms in terms of decreasing

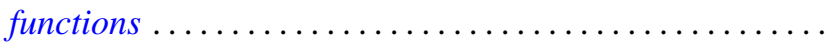

Stephen Scheinberg, Fatou's lemma in normed linear spaces

Ken Shaw, Whittaker constants for entire functions of several complex

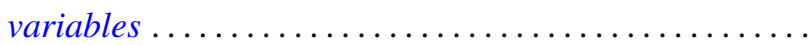

James DeWitt Stein, Two uniform boundedness theorems................ 251

$\mathrm{Li} \mathrm{Pi} \mathrm{Su,} \mathrm{Homomorphisms} \mathrm{of} \mathrm{near-rings} \mathrm{of} \mathrm{continuous} \mathrm{functions} \mathrm{.} \mathrm{.............} 261$

Stephen Willard, Functionally compact spaces, $C$-compact spaces and mappings of minimal Hausdorff spaces....................... 